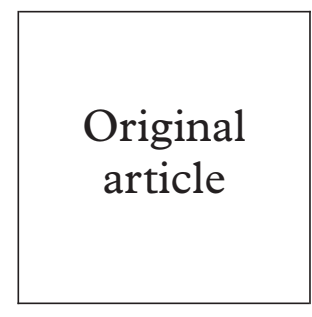

Original article

Sexually Transmitted Infections Research Centre, The University of Sydney, Marian Villa, Westmead

Hospital, Westmead, NSW 2145 Australia R L Tideman

C Marks

A Mindel

Centre for Virus Research Westmead Millennium Institute, Westmead Hospital, Westmead NSW 2145 Australia

J Taylor

C Seifert

A Cunningham

Department of Public Health and

Community Medicine, University of Sydney,

Sydney, NSW 2006,

Australia

G Berry

Department of Obstetrics and

Gynaecology,

Westmead Hospital,

Westmead, NSW 2145,

Australia

B Trudinger

Correspondence to:

Professor A Mindel

adrianm@

icpmr.wsahs.nsw.gov.au

Accepted for publication 26 June 2001

\title{
Sexual and demographic risk factors for herpes simplex type 1 and 2 in women attending an antenatal clinic
}

\author{
R L Tideman, J Taylor, C Marks, C Seifert, G Berry, B Trudinger, A Cunningham, \\ A Mindel
}

Objective: To establish risk factors for the presence of HSV-2 and HSV-1 infections in pregnant women.

Design, population, and setting: A prospective study of 3306 women attending the antenatal department Westmead Hospital, Sydney, between June 1995 and April 1998.

Methods: Women completed a self administered questionnaire to establish risk factors for the presence of HSV-2 and HSV-1. Sera were tested for antibodies to HSV-2 and HSV-1. Data were analysed using SPSS and SAS.

Main outcome measures: Seroprevalence of and risk factors for HSV-2 and HSV-1.

Results: 375 (11.3\% (95\% CI 10.3-12.5)) women were HSV-2 antibody positive. Increasing age, Asian country of birth, lower education level, public hospital status, confirmed genital herpes, a partner with genital herpes, early age of first sex, more than one lifetime sexual partner, and previous chlamydia infection were independently associated with HSV-2 seropositivity. Of 408 women tested for HSV-1 antibodies, 323 (79.2\% (95\% CI 74.9-83.0)) were positive. Oral herpes, oral blisters or sores, and being HSV-2 seropositive were independently associated with HSV-1 seropositive status. When the logistic regression model was rerun without HSV-2 status, parity of two or more and one or more sexual partners in the past 3 months were significant predictors of HSV-1 seropositivity.

Conclusions: The presence of antibodies to HSV-2 and HSV-1 is related to a number of sexual and demographic risk factors. Public health campaigns directed at encouraging young people to delay the onset of sexual activity and reduce the number of sexual partners need to be evaluated. However, the possible availability of an HSV-2 vaccine that is able to protect over $70 \%$ of women offers the best hope for control of genital herpes.

(Sex Transm Inf 2001;77:413-415)

Keywords: herpes simplex virus serology; demographic risk factors; sexual risk factors

\section{Introduction}

Genital herpes can be caused by herpes simplex virus type 1 (HSV-1) or HSV-2. However, about $50 \%$ of new cases of genital herpes are now due to HSV-1. Type specific serology has established that HSV infections are common and mostly asymptomatic. ${ }^{1}$ Risk factors for the acquisition of HSV-2 include age, sex, education level, number of sexual partners, age of coitarche, and a history of previous sexually transmitted infections (STIs). ${ }^{2-8}$ Some risk factors for the acquisition of $\mathrm{HSV}-1$ may also be sexually related. ${ }^{8}$

Identifying risk factors for HSV infection provides the opportunity to develop health promotion programmes based on primary prevention. Therefore, we sought to determine the risk factors for HSV-2 and HSV-1 infections in a group of women attending the antenatal clinic of a large teaching hospital in Sydney.

\section{Methods}

The study was conducted in the obstetric department at Westmead Hospital, Sydney, Australia, between June 1995 and April 1998. The Western Sydney Area Health Service research ethics committee approved the study. Written informed consent was obtained.
Participants completed a self administered questionnaire covering demographic details and risk factors for HSV infection such as age, age of coitarche, lifetime numbers of sexual partners, and a history of genital herpes.

Maternal and cord serum samples were collected to establish the HSV-2 seroprevalence and seroconversion rates during pregnancy. ${ }^{9}$ Samples were tested for HSV-2 antibodies using an indirect enzyme linked immunosorbent assay (ELISA) specific to glycoprotein G2 (gG2). ${ }^{10}$ The sensitivity and specificity of this assay is greater than $98 \%{ }^{10}$ and all equivocal results were resolved by western blot. ${ }^{11}$ If a maternal serum sample was not available the matching cord sample was used as the HSV-2 seroconversion rate during pregnancy for this population was very low ( 0.34 per 100 women years). ${ }^{9}$

Owing to financial constraints, a systematic sample of sera (one in eight) was tested to establish the HSV-1 seroprevalence. The Behring ELISA, which detects total antibody to HSV, was used to test HSV-2 seronegative samples. ${ }^{11}$ HSV-2 seropositive samples were tested using western blot. This technique was employed as an accurate, cheap, and labour efficient HSV-1 ELISA was not available. 
Table 1 Crude and adjusted odds ratios for demographic and behavioural variables associated with HSV 2 antibody status using logistic regression analysis

\begin{tabular}{|c|c|c|c|c|}
\hline Variable & No tested & $\% H S V-2^{*}$ & Crude OR $+(95 \%$ CI $) \neq$ & Adjusted 5 OR $†(95 \%$ CI $) \neq$ \\
\hline \multicolumn{4}{|l|}{ Age } & $\chi_{4}^{2}=56.29, \mathrm{p}=0.0001$ \\
\hline $15-19$ & 161 & 4.3 & $0.4(0.19-0.91)$ & $0.4(0.16-0.85)$ \\
\hline $20-24$ & 747 & 8.3 & $0.8(0.60-1.15)$ & $0.8(0.52-1.09)$ \\
\hline $25-29$ & 1164 & 9.7 & Reference group & 1.0 \\
\hline $30-34$ & 831 & 13.8 & $1.5(1.12-1.94)$ & $1.7(1.22-2.31)$ \\
\hline $35+$ & 366 & 19.9 & $2.3(1.66-3.15)$ & $3.0(2.08-4.44)$ \\
\hline \multicolumn{4}{|l|}{ Country of birth } & $\chi_{8}^{2}=17.89, \mathrm{p}=0.022$ \\
\hline Australia and NZ & 2082 & 11.5 & Reference group & 1.0 \\
\hline Polynesia & 93 & 14.0 & $1.3(0.69-2.31)$ & $2.2(1.04-4.51)$ \\
\hline $\mathrm{N}$ and SE Asia & 327 & 12.2 & $1.1(0.76-1.55)$ & $2.2(1.37-3.55)$ \\
\hline Southern Asia & 156 & 5.4 & $0.4(0.20-0.87)$ & $1.1(0.48-2.57)$ \\
\hline Africa & 64 & 3.1 & $0.3(0.06-1.03)$ & $0.2(0.03-1.65)$ \\
\hline Middle East & 207 & 10.1 & $0.8(0.48-1.28)$ & $1.3(0.75-2.41)$ \\
\hline Europe & 225 & 20.9 & $1.6(1.13-2.36)$ & $1.3(0.87-2.08)$ \\
\hline $\mathrm{N}$ America & 19 & 5.3 & $0.4(0.06-3.25)$ & $0.5(0.07-4.38)$ \\
\hline S America & 63 & 12.7 & $1.1(0.53-2.41)$ & $1.7(0.72-3.82)$ \\
\hline \multicolumn{4}{|l|}{ Education level } & $\chi_{3}^{2}=13.06, p=0.0045$ \\
\hline Primary & 110 & 17.3 & $2.0(1.18-3.36)$ & $2.0(1.05-3.72)$ \\
\hline Year 10 & 1039 & 14.4 & $1.6(1.26-2.05)$ & $1.5(1.15-2.07)$ \\
\hline Year 12 & 641 & 9.7 & $1.0(0.75-1.40)$ & $0.9(0.64-1.34)$ \\
\hline Tertiary & 1454 & 9.6 & Reference group & 1.0 \\
\hline \multicolumn{4}{|l|}{ Hospital status } & $\chi_{3}^{2}=10.78, p=0.0010$ \\
\hline Private & 384 & 6.3 & Reference group & 1.0 \\
\hline Public & 2862 & 13.5 & $2.0(1.32-3.11)$ & $2.2(1.39-3.65)$ \\
\hline \multicolumn{4}{|c|}{ Confirmed genital herpes } & $\chi_{1}^{2}=45.23, p=0.0001$ \\
\hline No & 3147 & 10.3 & Reference group & 1.0 \\
\hline Yes & 99 & 46.5 & $7.5(5.00-11.37)$ & $5.9(3.50-9.82)$ \\
\hline \multicolumn{4}{|c|}{ Partner with genital herpes } & $\chi_{1}^{2}=17.22, p=0.0001$ \\
\hline No & 3142 & 10.6 & Reference group & 1.0 \\
\hline Yes & 71 & 45.1 & $6.9(4.28-11.20)$ & $3.6(1.97-6.63)$ \\
\hline \multicolumn{4}{|l|}{ Age at first sex } & $\chi_{3}^{2}=11.32, p=0.010$ \\
\hline$<15$ & 377 & 14.6 & $1.2(0.84-1.59)$ & $1.0(0.67-1.44)$ \\
\hline $16-19$ & 1674 & 12.8 & Reference group & 1.0 \\
\hline $20-24$ & 737 & 8.5 & $0.6(0.47-0.85)$ & $0.8(0.58-1.20)$ \\
\hline$>24$ & 342 & 5.6 & $0.4(0.25-0.65)$ & $0.3(0.18-0.63)$ \\
\hline \multicolumn{4}{|c|}{ Lifetime number of sexual partners } & $\chi_{1}^{2}=17.23, \mathrm{p}=0.0001$ \\
\hline One & 1308 & 6.1 & Reference group & 1.0 \\
\hline$>$ one & 1749 & 14.6 & $2.6(2.02-3.41)$ & $2.1(1.46-2.89)$ \\
\hline \multicolumn{4}{|c|}{ History of chlamydia } & $\chi_{1}^{2}=13.53, p=0.0002$ \\
\hline No & 3151 & 10.8 & Reference group & 1.0 \\
\hline Yes & 82 & 29.3 & $3.4(2.09-5.56)$ & $2.9(1.64-5.02)$ \\
\hline
\end{tabular}

*Percentage of those tested who were HSV-2 seropositive (\%HSV-2).

†Odds ratio.

$\$ 95 \%$ confidence interval.

\Adjusted for all the factors in tables 1 and 2 .

Table 2 Crude and adjusted odds ratios for variables significantly associated with HSV 1 seropositive status using logistic regression analysis

\begin{tabular}{|c|c|c|c|c|}
\hline Variable & No tested & $\% H S V-1^{*}$ & Crude OR† $(95 \%$ CI $) \neq$ & Adjusted OR† $(95 \% \mathrm{CI}) \neq$ \\
\hline \multicolumn{5}{|c|}{ (A) Maternal HSV-2 status in logistic regression model } \\
\hline \multicolumn{4}{|c|}{ History of oral herpes } & $\chi_{1}^{2}=4.43, p=0.035$ \\
\hline No & 293 & 72.0 & reference group & 1.0 \\
\hline Yes & 108 & 98.1 & $20.6(4.97-85.38)$ & $5.7(1.13-28.90)$ \\
\hline \multicolumn{4}{|c|}{ History of oral blisters/sores } & $\chi_{1}^{2}=7.73, p=0.005$ \\
\hline No & 269 & 70.3 & reference group & 1.0 \\
\hline Yes & 133 & 97.0 & $13.7(4.88-38.19)$ & $5.3(1.64-17.42)$ \\
\hline \multicolumn{4}{|c|}{ HSV-2 seropositive } & $\chi_{1}^{2}=7.19, \mathrm{p}=0.007$ \\
\hline Negative & 352 & 76.4 & reference group & 1.0 \\
\hline Positive & 54 & 96.3 & $8.0(1.91-33.64)$ & $7.3(1.71-31.39)$ \\
\hline \multicolumn{5}{|c|}{ (B) Maternal HSV-2 status removed from the logistic regression model } \\
\hline \multicolumn{4}{|l|}{ Parity } & $\chi_{2}^{2}=6.52, p=0.038$ \\
\hline None & 176 & 72.7 & Reference group & 1.0 \\
\hline One & 142 & 81.0 & $1.6(0.94-2.73)$ & $1.5(0.82-2.59)$ \\
\hline Two or more & 90 & 88.9 & $3.0(1.44-6.27)$ & $2.7(1.23-5.95)$ \\
\hline \multicolumn{4}{|c|}{ Number of partners in the past 3 months } & $\chi_{1}^{2}=4.40, \mathrm{p}=0.036$ \\
\hline None & 9 & 55.6 & Reference group & 1.0 \\
\hline One or more & 380 & 79.2 & $3.0(0.80-11.62)$ & $5.7(1.12-29.41)$ \\
\hline \multicolumn{4}{|c|}{ History of oral blisters/sores } & $\chi_{1}^{2}=25.66, p=0.0001$ \\
\hline No & 269 & 70.3 & Reference group & 1.0 \\
\hline Yes & 133 & 97.0 & $13.7(4.88-38.19)$ & $15.4(5.34-44.41)$ \\
\hline
\end{tabular}

${ }^{\star}$ Percentage of those tested who were HSV-1 seropositive (\%HSV-1).

†Odds ratio.

$\$ 95 \%$ confidence interval.

Questionnaire responses and serology results were entered onto a database. Using SPSS and SAS, univariate and multiple logistic regression analyses were performed to establish which demographic and behavioural factors, were associated with HSV serostatus.

\section{Results}

A total of 3706 women were recruited. However, 400 were excluded as no serum sample was available. The reasons for missing sera included mislaid samples, sample never taken, or sample taken at a private laboratory and not available for testing. In all, 3306 maternal or cord HSV-2 results and 408 maternal HSV-1 results were available for analysis.

RISK FACTORS FOR HSV-2 SEROSTATUS

Three hundred and seventy five of the 3306 $(11.3 \%$ 95\% CI 10.3-12.5\%) women were HSV-2 antibody positive. A large number of demographic and behavioural risk factors were associated with HSV-2 seropositivity. These were country of birth $(\mathrm{COB})(\mathrm{p}<0.01)$, education level, age, whether they were public or private patients, previous genital herpes, a partner with genital herpes, lifetime number of sexual partners, age at coitarche, number of pregnancies, number of live births, history of chlamydia, history of human papillomavirus infection (all $\mathrm{p}=0.001$ ), and history of syphilis $(\mathrm{p}<0.04)$. Although the lifetime number of partners is shown as a dichotomy, one and more than one, further analysis showed a trend of increasing risk with an odds ratio for more than 20 partners of 6.8 (95\% CI 3.8-12.1).

On the basis of statistical significance $(\mathrm{p}<0.05)$ and perceived clinical importance 11 variables assessed using univariate analysis were entered into the multiple logistic regression model. Gravidity and history of warts were subsequently excluded as they were found to be significant confounders. The final model contained nine variables. The results are summarised in table 1. Increasing age, a north Asian or South East Asian country of birth, lower education level, public hospital status, confirmed genital herpes, a partner with genital herpes, early age of coitarche more than one lifetime partner, and previous genital chlamydia infection were the predictors significantly associated $(\mathrm{p}<0.05)$ with HSV-2 seropositive status using multiple logistic regression analysis (table 1).

RISK FACTORS FOR HSV-1 SEROSTATUS

Three hundred and twenty three of the 408 (79.2\% (95\% CI 74.9-83.0)) serum samples available for testing were HSV-1 positive. On univariate analysis, country of birth, previous oral herpes, history of oral blisters or sores, HSV-2 serostatus, parity, and gravidity were significantly associated with HSV-1 serostatus. Multiple logistic regression analysis found that previous oral herpes, a history of oral blisters or sores, and HSV-2 seropositive status were the only independent $(\mathrm{p}<0.05)$ predictors of HSV-1 serostatus. However, when the logistic regression model was rerun without HSV-2 status, parity of two or more and one or more sexual partners in the past 3 months, in addition to a history of oral blisters or sores, were significant predictors of HSV-1 seropositivity (table 2). 


\section{Discussion}

This study has shown that in women there are many demographic and sexual risk factors for the presence of HSV-2. The demographic risk factors that were independently associated with HSV-2 were north Asian and South East Asian country of birth, lower education level, public hospital status and increasing age. The sexual risk factors were early age at coitarche, more than one lifetime sexual partner, having a partner with genital herpes, and previous chlamydia infection. In addition, women with a history of genital herpes were almost six times more likely to be HSV-2 seropositive than those who were not.

These findings confirm the importance of sexual risk factors for the acquisition of HSV-2. In our study, women with more than one lifetime sexual partner were twice as likely to have HSV-2 antibodies than those with one and women who delayed coitarche until they were 24 or over, were $70 \%$ less likely to have antibodies to HSV-2 than those who commenced sexual activity at 15 or younger. Age, fewer years of education, and whether the patient was public or private (an indirect measure of socioeconomic status) have all been shown in previous studies and confirmed in our study to be independent risk factors for HSV-2 infection. ${ }^{10}$

In contrast with HSV-2 infection, our study revealed a small number of risk factors (previous oral herpes, previous oral blisters or sores, and HSV-2 seropositivity) for HSV-1 seropositivity. When HSV-2 was removed from the multiple logistic regression model, HSV-1 was found to be associated with increasing parity and one or more sexual partners in the past 3 months, suggesting that HSV-1 infection may be related to sexual behaviour. This is the first evidence that both HSV-1 and HSV-2 are associated with the number of sexual partners.

This study has identified two behavioural risk factors for HSV-2 infection that could be important in mounting an effective health promotion campaign, the age of coitarche, and the number of sexual partners. Encouraging young people to delay coitarche and to limit the number of sexual partners may be a difficult message to sell but the potential health gains, especially for women less than 20 years old, are considerable. Studies to develop innovative health promotion programmes involving young people will be essential to ensure success. The possible availability of an HSV-2 vaccine that is able to protect over $70 \%$ of women offers the best hope for control of genital herpes. ${ }^{12}$

This study was funded by grants from the Ramaciotti Foundation and the Australian National Health and Medical Research Council (954001). We would like to thank Dr Catherine O'Connor for her assistance at the beginning of the study, Jane Griffith, the nursing and midwifery staff in the antenatal clinics, Jude Page, Katherine Wagner, Jocelyn Ling, and Stuart Gilmore for their help in recruiting patients, Mr Con Tsiavos from the for their help in recruiting patients, Mr Con Tsiavos from the Information Services Division at Westmead Hospital for as

nce in data retrieval, and Stig Jeanson for the gG2 antigen. Contributors: RLT was involved in the day to day coordination, statistical analysis, and interpretation and manuscript preparation; JT did the HSV serology, data retrieval, and manuscript review; CM did the data entry and review, statistical analysis, and manuscript review; CS did the HSV serology, data retrieval, and manuscript review; GB did the statistical analysis, review and interpretation, and manuscript review; BT was involved in study design and manuscript review; AC was involved in the initial idea, study design, and manuscript review; AM was involved in the initial idea, study design, overall coordination, and manuscript review.

1 Mindel A. Genital herpes-how much of a public-health problem? Lancet 1998;351(suppl III): 16-18.

2 Uribe-Salas F, Hernandez-Avila M, Juarez-Figueroa L, et al. Risk factors for herpes simplex virus type 2 infection among female commercial sex workers in Mexico City. Int f STD AIDS 1999;10:105-11.

3 Cowan F, Johnson AM, Ashley R, et al. Antibody to herpes simplex virus type 2 as serological marker of sexual lifestyle in populations. BMF 1994;309:1325-9.

4 Cunningham AL, Lee FK, Ho DW, et al. Herpes simplex virus type 2 antibody in patients attending antenatal or STD clinics. Med f Aust 1993;158:525-8.

5 Wald A, Koutsky L, Ashley RL, et al. Genital herpes in a primary care clinic: demographic and sexual correlates of herpes simplex type 2 infections. Sex Transm Dis 1997;24:14955 .

6 Johnson RE, Nahmias AJ, Magder LS, et al. A seroepidemiologic survey of the prevalence of herpes simplex virus type 2 infection in the United States. $N$ Engl $f$ Med 1989;321:7-12.

7 Stavraky KM, Rawls WE, Chiavetta J, et al. Sexual and socioeconomic factors affecting the risk of past infections with herpes simplex virus type 2. Am F Epidemiol 1983;118: 109-21.

8 Gibson JJ, Hornung CA, Alexander GR, et al. A cross-sectional study of herpes simplex virus types 1 and 2 in college students: occurrence and tion. F Infect Dis 1990;162:306-12.

9 Mindel A, Taylor J, Tideman RL, et al. Neonatal herpes prevention: a minor public health problem in some prevention: a minor public health problem
communities. Sex Transm Inf 2000;76:287-91.

10 Ho DW, Field PR, Sjogren-Jansson E, et al. Indirect ELISA for the detection of HSV-2 specific IgG and IgM antibodies with glycoprotein G (gG-2). F Virol Methods 1992;36: ies with $249-64$.

11 Ho DW, Field PR, Irving WL, et al. Detection of immunoglobulin $M$ antibodies to glycoprotein G-2 by western blot (immunoblot) for diagnosis of initial herpes simplex genital type 2 infections. F Clin Microbiol 1993;31: 3157-64.

12 Spruance SL, and the Smithkline Beecham (SB) Herpes Vaccine Efficacy Group. Gender-specific efficacy of a prophylactic sBASA-adjuvanted GD2 subunit vaccine against genital disease (GHD): results of two clinical efficacy trials. Abstract, ICAAC, Toronto, 2000. 